\title{
Structural organization of the Golgi apparatus
}

Martin Lowe

Faculty of Life Sciences, University of Manchester, The Michael Smith Building, Oxford Road, Manchester, M13 9PT, U.K.

Tel: 0161-275-5387. Fax: 0161-275-1505. E-mail: martin.lowe@manchester.ac.uk

Word count: 3771 (excluding references and figure legends) 
The Golgi apparatus is a universal feature of eukaryotes, carrying out the key functions of processing, sorting and trafficking of newly synthesized membrane and secretory proteins. The Golgi apparatus has a clearly defined structure, comprising stacks of flattened cisternal membranes that in vertebrates are connected to form a ribbon. How this structure is maintained and how it relates to the functions of the Golgi apparatus has long been an area of interest. In this review I describe recent progress in the identification and characterisation of the molecular machinery that together help generate the characteristic organization of this organelle. 


\section{Introduction}

Since its discovery over 100 years ago the Golgi apparatus has fascinated biologists [1-4]. This organelle lies at the heart of the secretory pathway and performs numerous functions including modification of secretory cargoes and their sorting and delivery to various destinations, which may be inside or outside the cell. The Golgi apparatus has a characteristic structure that is conserved amongst nearly all eukaryotes, comprising flattened membrane discs called cisternae that are layered on top of each other to generate the Golgi stack. Most 'lower' eukaryotes contain one or more discrete Golgi stacks per cell, while in vertebrates the stacks are typically linked to form the Golgi ribbon, a single copy structure usually found next to the centrosome. Despite our long acquaintance with the Golgi apparatus, there remain many questions regarding how this organelle is assembled and how it carries out its varied functions. In this review I will focus on the structural organization of the Golgi apparatus, highlighting recent progress in the identification of molecular components and mechanisms that help explain how the Golgi achieves its functional form in different organisms.

\section{How to make a Golgi stack?}

In almost all eukaryotes the Golgi apparatus is made up of stacked cisternae. The cisternae contain resident enzymes that sequentially process cargo proteins and lipids, most notably at the level of glycosylation, as they move across the stack in a vectorial fashion. The mechanism by which cargo is transported across the Golgi stack remains contentious, but most available evidence supports the idea of cisternal maturation (for recent reviews see $[5,6])$. In this model, cargo is retained within cisternae that progressively mature as they migrate across the stack in a cis to trans direction. The maturation of cisternae is brought about by the continual recycling of Golgi enzymes in retrograde vesicles that bud from the 
rims of 'later' cisternae and fuse with adjacent 'earlier' cisternae. Alternative models for transport involve anterograde trafficking in intra-Golgi vesicles [7], or transport through tubular continuities connecting different cisternae within the Golgi stack [8-10]. Although the evidence for these transport mechanisms is less compelling, it remains possible they prevail for certain cargoes or cell types, perhaps in co-existence with cisternal maturation.

\section{GRASPs as Golgi stacking factors}

Regardless of how cargo transits the Golgi stack, it is clear that mechanisms must exist to generate and maintain the identity and structural integrity of this compartment. In line with this idea, early studies showed the existence of proteinaceous structures cross-linking adjacent Golgi cisternae [11-13]. Attractive candidates for these cross-linking proteins are the GRASPs (Golgi Reassembly And Stacking Proteins). There are two GRASPs in vertebrates, termed GRASP65 and 55, that localise to cis and medial cisternae respectively, while 'lower' eukaryotes have only a single GRASP protein [14-18]. The GRASPs are enriched in a detergent resistant matrix fraction isolated from purified Golgi membranes [14,19]. Using an in vitro assay for post-mitotic assembly of rat liver Golgi stacks, it was demonstrated that both GRASP65 and 55 participate in the stacking of cisternae, supporting their role as Golgi stacking factors $[14,15]$. Initial RNA interference experiments supported this conclusion since depletion of GRASP65 gave a partial loss of stacking in vivo [20]. However, subsequent studies reported that depletion of GRASP65 or 55 appeared to have little effect on Golgi stacking, instead leading to a loss of Golgi ribbon continuity and prompting the hypothesis that GRASPs laterally link adjacent cisternae in the Golgi ribbon rather than stacking cisternae $[21,22]$. A recent study has attempted to resolve these discrepancies by combining depletion of the mammalian GRASPs with high resolution quantitative electron 
microscopy analysis of Golgi structure [23**]. Using this approach it was shown that depletion of either GRASP alone gives a minor but consistent reduction in the number of cisternae within a stack, while depletion of both completely abolishes stack formation, resulting in the generation of vesiculated Golgi remnants. Re-expression of either GRASP can partially rescue this phenotype. These results strongly support the idea that the vertebrate GRASPs have complementary and essential roles in stacking Golgi cisternae.

How might GRASPs contribute to Golgi stacking? Vertebrate GRASPs are able to form homo-oligomers that can cross-link latex beads in vitro [23**,24,25*], or mitochondria in vivo when targeted to this organelle [26*]. GRASP trans-oligomer formation would therefore seem a good way to cross-link Golgi membranes during cisternal stacking. GRASP oligomerization is mediated by its first PDZ domain, that alone is sufficient to cross-link membranes $\left[25^{*}, 26^{*}\right]$. For this to occur efficiently, the PDZ domain needs to be correctly oriented, likely through a dual anchoring mechanism in which the PDZ domain is flanked by upstream and downstream membrane attachment sites [27]. The first of these is an Nterminal myristoylation site, while the second attachment site corresponds to the second PDZ domain, which in GRASP65 stably binds the extreme C-terminus of the golgin GM130 $[14,27,28]$. Like other golgins, GM130 is a predicted elongated coiled-coil protein that extends from the membrane. GM130 binds the vesicle docking protein p115 at its Nterminus, and has been implicated in several membrane tethering events at the cis-Golgi (reviewed in [29]). In vitro studies indicate that GM130-mediated tethering is required for cisternal stacking during Golgi assembly, acting before GRASP65 [30]. We can therefore envisage a model in which GM130 initiates long-range tethering followed by trans- 
oligomerization of GRASPs to form stable cross-links between apposing Golgi cisternae, resulting in stacking (Fig 1).

\section{Additional functions for GRASPs}

When it comes to Golgi stacking GRASPs cannot be the whole story, however. Loss of the single GRASP in the fruit fly Drosophila melanogaster has a minor effect on Golgi stacking [17], while in the budding yeast Saccharomyces cerevisiae, the cisternae are largely unstacked despite the presence of a GRASP protein known as Grh1p [16,18]. On top of that, plants have stacked Golgi cisternae yet appear to lack a GRASP altogether [31**,32]. These observations suggest that additional factors can stack Golgi cisternae (discussed in more detail below), and that GRASPs can have other functions in addition to cisternal stacking. As mentioned above, GRASPs are involved in laterally linking Golgi cisternae to form a ribbon in vertebrates $[21,22]$ (Fig 1). GRASPs may also play role in the tethering of transport vesicles, possibly via anchoring of GM130. In support of this idea, budding yeast Grh1p and its partner Bug1p, a GM130 homologue, genetically interact with ER to Golgi trafficking components [16]. GRASPs can also participate in the trafficking of certain cargoes through direct binding to these proteins as they enter and transit the Golgi stack $\left[33^{*}, 34^{*}\right]$. Finally, several recent studies have revealed a role for GRASPs in unconventional secretion i.e. that avoiding the typical secretory route through the Golgi apparatus [35,36]. During Drosophila melanogaster development, GRASP relocates to the plasma membrane where it is important for the delivery of integrins during epithelial remodelling [36]. Here, transport intermediates are thought to bypass the Golgi and dock with GRASP at the cell surface instead of the cisGolgi. In the slime mould Dictyostelium discoideum and budding yeasts, GRASP is required for the unconventional secretion of an acyl coenzyme binding protein, which occurs via an 
autophagosomal intermediate $\left[35,37 *, 38^{*}\right]$. How GRASP contributes to this process is currently unclear, but it is interesting to note that other Golgi proteins have been implicated in autophagosome formation, suggesting that the Golgi apparatus plays an important role [3941].

GRASPs are present in all eukaryotes, with the apparent exception of plants, pointing to an important ancestral function for these proteins [42]. The varied observations described above are consistent with GRASPs functioning to link membranes together. We can speculate that this is a conserved function of GRASPs, one that has been adapted for different purposes depending upon the organism, cell type, or stage in life cycle. GRASP-mediated membrane linking could therefore be used for cisternal stacking, vesicular traffic, linking the Golgi ribbon, or autophagosome formation. Additional roles for GRASPs, such as binding to cargo during Golgi trafficking, as occurs in vertebrates, are likely to have appeared later in evolution. Certain eukaryotes such as plants are able to stack their Golgi without using a GRASP. Either stacking in these organisms uses a fundamentally different mechanism to the GRASP-dependent cross-linking seen in vertebrates, or a similar mechanism is employed using distinct but functionally equivalent proteins to the GRASPs.

\section{Microtubules and building the Golgi ribbon}

In most vertebrate cells individual Golgi stacks are laterally connected to form a ribbon [43]. The Golgi ribbon is often located adjacent to the nucleus around the centrosome, implying a functional link with the microtubule cytoskeleton. Indeed it has been known for many years that an intact microtubule network and the minus end-directed microtubule motor cytoplasmic dynein are required to maintain an intact Golgi ribbon [44]. Loss of either results 
in the dispersion of Golgi stacks, which accumulate next to ER exit sites [45]. Dynein couples with ER to Golgi carriers and drives their movement into the cell centre, where homotypic fusion events laterally connect cisternae to form the Golgi ribbon. Maintenance of the ribbon is dependent upon the continued presence of both microtubules and dynein motor activity.

Recent studies have revealed that the vertebrate Golgi apparatus can function as a microtubule organizer in its own right, able to nucleate microtubules via a pool of $\gamma$-tubulin localized to this compartment $\left[46,47^{* *}, 48^{*}\right]$. Golgi-derived microtubules form an asymmetric network that preferentially extends towards the leading edge of migrating cells, that is important for the polarized delivery of Golgi-derived carriers to this region of the plasma membrane $\left[47^{* *}, 48^{*}, 49^{*}\right]$ (Fig 2). Golgi-derived microtubules are more extensively modified than those nucleated at the centrosome, which is likely important for their altered dynamics and interaction with trafficking intermediates, such as those targeted to the leading edge of cells $\left[48^{*}\right]$. The formation of microtubules at the trans-Golgi is dependent upon the regulator CLASP, which is recruited to the membrane through interaction with the golgin GCC185 [47**]. It also requires active dynein, as is the case for centrosomal microtubules. Microtubules can also form at the cis-Golgi via a distinct mechanism involving AKAP450, a known interactor of the $\gamma$-tubulun ring complex found at the centrosome [48*]. AKAP450 is recruited to the cis-Golgi through binding to GM130, and microtubules nucleated by this complex are important for polarized secretion.

In addition to playing a role in polarized trafficking, CLASP-dependent Golgi-nucleated microtubules are also required for assembly of the Golgi ribbon [49*]. During Golgi 
assembly, they act in a 'search and capture' fashion to bring together individual Golgi stacks, which are subsequently transported from the periphery to the cell centre via dynein-mediated trafficking along the centrosomal microtubule array. Golgi ribbon formation therefore requires two sets of independently nucleated microtubules acting in concert (Fig 2). The Golgi-derived microtubules are also important for Golgi ribbon maintenance, most likely by keeping stacks in close enough proximity for linking by tubular connections. In geometric terms it makes sense for the tubular connections between stacks within the Golgi ribbon to use the tangentially arranged Golgi-derived microtubules rather than those from the central centrosomally-nucleated array.

\section{The Golgi matrix}

Biochemical and morphological studies support the existence of a Golgi matrix for scaffolding Golgi assembly and maintaining its structural integrity $\left[11-13,19,31^{* *}, 50\right]$. EM studies indicate a so-called 'ribosome exclusion zone' surrounding the Golgi apparatus, suggesting that the matrix extends into the cytoplasm surrounding the organelle $[31 * *, 51,52]$

(Fig 3). The composition of this surrounding matrix remains to be defined, but excellent candidates are members of the golgin family of coiled-coil proteins, of which there are at least 20 types in vertebrates (reviewed in [29]). The predominant function of golgins appears to be in the tethering or long-range attachment of membranes, a process coordinated by Rab GTPases. Tethering can be followed by membrane fusion, as is the case for transport intermediates or in linking of the Golgi ribbon, or not, as in the case of cisternal stacking. Different golgins are thought to participate in different tethering reactions that include tethering intra-Golgi vesicles or transport carriers arriving from other compartments to Golgi cisternae, linking cisternae to form a ribbon, or contributing to cisternal stacking. Some 
golgins also link the Golgi apparatus to the cytoskeleton. Given their predicted elongated structure, and the fact that most golgins are anchored at the Golgi membrane by one end, we can envisage a scenario in which the Golgi is coated with a network of protruding tentacles that are able to tether incoming transport intermediates over a relatively long range $[29,53]$. In the case of intra-Golgi transport, it is tempting to speculate that during their formation, or very soon afterwards, the vesicles become tethered to adjacent cisternae via golgin-mediated attachments to limit their diffusion and ensure highly efficient transport within the stack. In support of this idea, Golgi stacks are surrounded by numerous vesicles within close proximity $[43,54]$ (Fig 3). Detailed inspection of intra-Golgi vesicles shows the presence of proteinaceous rod-like extensions protruding from their surface that may well correspond to golgins [55].

According to the cisternal maturation model Golgi cisternae will move through the stack as they mature. This invokes a highly dynamic matrix, in which contacts between cisternae need to be broken and reformed as the cisternae progress through the stack. One of the few studies to look at Golgi matrix protein dynamics has shown that GFP-tagged GRASP65 is dynamically associated with the Golgi apparatus, undergoing rapid exchange with the cytosol [56]. This is consistent with the notion of a dynamic matrix, but whether it applies to other matrix components remains to be shown. It is also unclear how remodelling of the Golgi matrix would take place. Attractive candidates for "matrix remodellers" are the Rab GTPases, which interact with most if not all golgins [29,57]. Prominent among these Rab GTPases are Rab 1, 2, 6 and 33b. The status of Rab activation is under tight control by GEFs and GAPs, allowing a high degree of spatial and temporal regulation [58]. In addition to recruiting some golgins to the membrane, Rab binding is likely important for tethering to 
Rab-coated transport vesicles or membrane domains, and it may play a role in controlling conformation and availability of golgin tethers.

In mammalian cells, remodelling of the Golgi matrix has been observed during polarisation for cell migration or upon mitotic entry. Phosphorylation of GRASP65 by ERK (extracellular signal-regulated kinase) is required for cisternal unstacking and Golgi repositioning towards the leading edge in migrating fibroblasts [59*]. When vertebrate cells enter mitosis, phosphorylation of many matrix proteins including the GRASPs and golgins takes place, allowing complete disassembly of the Golgi apparatus and its equal partitioning into the daughter cells [60]. Whether phosphorylation of Golgi matrix proteins occurs in nonvertebrates is unclear. It is unlikely to occur during mitosis in these species, which do not fragment their Golgi apparatus during cell division. However, it remains possible phosphorylation or other post-translational modifications such as arginine methylation may regulate aspects of Golg matrix behaviour in all eukaryotes [61].

\section{Actin and actin binding proteins}

Actin and numerous actin binding proteins have been localized to the Golgi apparatus, and many of these have been shown to be important for Golgi function (reviewed in $[62,63])$. It is clear that actin-based myosin motors are important for the movement of Golgi stacks in lower eukaryotes, while in vertebrates this is accomplished by microtubule-associated motor proteins. However, myosin motors do play a role in Golgi organization in higher eukaryotes [64]. A recent example is provided by the Golgi protein GOLPH3 (also known as GMx33 or Vps74p in yeast) which binds to myosin 18A [65]. The interaction between GOLPH3 and myosin $18 \mathrm{~A}$ is required for extension of the Golgi ribbon and the formation of transport 
carriers, most likely by providing tension across the surface of the Golgi membrane. Analysis of the Drosophila melanogaster Golgi apparatus has shown that it duplicates and exist as a paired structure during the G1/S phase of the cell cycle [66]. Pairing of the Golgi stacks is dependent upon a subset of actin filaments that in turn require the regulatory proteins Abi and Scar/WAVE for their polymerization. Interestingly, this organization also seems to apply to mammalian cells, with pairing of stacks occurring within the context of the Golgi ribbon, suggesting a conserved role for this process in Golgi duplication [66].

\section{Golgi structure versus function}

A question that has long been asked is why does the Golgi exist as stacked cisternae in most eukaryotes? Several unicellular eukaryotes have lost Golgi stacked cisternal morphology during evolution, yet retain efficient trafficking through this compartment [42]. Furthermore, during certain stages of Drosophila melanogaster development the Golgi is not stacked yet secretion takes place [67]. While there is no definitive explanation for Golgi stacking, several possible reasons exist. The compartmentalization of the Golgi into cisternae should promote efficient cargo processing by ensuring sequential exposure of cargo to the modifying enzymes, with optimum enzyme levels and environments for the enzymatic reactions present in each cisternae. It is interesting to note that certain species lacking Golgi stacks have fewer glycosylases than relatives with a stacked Golgi, suggesting that a correlation between stacking and the extent of glycosylation may exist (discussed in [42]). The stacked Golgi also allows for iterative sorting of cargo, as originally suggested by the distillation hypothesis of James Rothman [68]. Thus, stacking should improve efficiency of cargo sorting. Another potential advantage conferred by Golgi stacking is improved efficiency of trafficking between cisternae, which may be of relevance to larger cells. Holding cisternae together 
means that there is little physical distance for intra-Golgi vesicles to travel, while continual attachment to the matrix would ensure efficient capture by preventing diffusion into the surrounding cytoplasm. Stacking may also offer a means to regulate flux through the secretory pathway since loss of stacking can stimulate the rate of intra-Golgi vesicle formation, at least in vitro [69]. By cross-linking cisternae, stacking appears to limit the amount of membrane available for incorporation into transport vesicles.

The Golgi ribbon is only found in vertebrates, a consequence of the intimate association between Golgi membranes and microtubules that exists in these higher eukaryotes [44]. Loss of the Golgi ribbon has only a minor effect on the rate of secretory trafficking. It has been proposed that Golgi ribbon formation is required for optimum glycosylation of cargo [21]; however it is clear that organisms that lack the ribbon such as plants are able to efficiently glycosylate large amounts of cargo. Perhaps the uniform glycosylation of cargo afforded by the Golgi ribbon is especially important in vertebrate cells for reasons that are currently unclear. Formation of the Golgi ribbon is clearly important for directed secretion, most notably to the leading edge of migrating cells [70]. Interestingly, when ribbon remodelling is prevented, orientation of the centrosome towards the leading edge is also prevented, indicating that the Golgi ribbon can determine centrosome positioning [59*]. Thus the vertebrate Golgi is dependent upon the centrosome for its positioning but the converse is also true.

Golgi ribbon status is monitored in vertebrate cells as they pass through the G2 phase of the cell cycle. Typically the Golgi stacks are unlinked in the G2 phase as a prelude to complete disassembly that occurs when the cells enter mitosis. If unlinking of stacks fails to occur, 
cells will not enter mitosis, indicating the existence of a cell cycle 'Golgi checkpoint' $[71,72]$. The underlying mechanisms of this checkpoint remain to be discovered, but are likely to involve cross-talk between the Golgi apparatus and the centrosome. Interestingly GM130 has recently been shown to have a role in centrosome integrity during interphase, indicating that such a cross-talk can exist [73].

The structure of the Golgi apparatus is inextricably linked to membrane traffic. Changing rates of trafficking into or out of the Golgi has profound effects on its structure (reviewed in [74]). Many of the structural proteins described above are also important players in membrane traffic. The golgins for example link membranes during trafficking, and this linking is also important for maintenance of Golgi structure [29]. Thus we should not think of the Golgi as a static structure, but rather one that is in dynamic equilibrium with the membrane and cargo flowing through it. The ability of the Golgi to undergo reversible disassembly under a number of conditions indicates it has a high propensity to self-organise, with matrix proteins, cytoskeletal and trafficking factors all contributing to the generation of an organized and functional Golgi apparatus.

\section{Concluding remarks}

In this review I have described molecular players and associated mechanisms that are important for the generation of the characteristic organization of the Golgi apparatus. Although much progress has been made in recent years a number of outstanding issues remain. Key among these are how cisternal stacking occurs. In vertebrates GRASP oligomerization seems able to do this, yet there is a poor correlation between GRASPs and stacking in other organisms. If GRASPs are not involved in cisternal stacking in these 
species, then what is? Perhaps golgins play a role, possibly in conjunction with additional factors that stabilise tethers and promote closer apposition of cisternae. A possible way to address this would be to reconstitute stacking using purified components, similar to the recent demonstration of vesicle tethering by the golgin GMAP210 [75**]. High resolution imaging combined with immunolabelling should also prove informative, since we would predict that stacking proteins should reside within the intercisternal spaces. Combining imaging with knockout studies in genetically tractable organisms is also likely to be of benefit. Another important question relates to the functional importance of golgins. Loss of a single golgin often yields a mild phenotype or none at all, suggesting a high level of redundancy amongst these proteins. However, recent studies in animal models suggest this might not be the case for all golgins $\left[76^{*}, 77^{*}\right]$. Instead it appears that golgin-mediated tethering is important for maintaining optimum Golgi function required for correct cargo processing and trafficking, which in the context of a whole organism is important for normal development and physiology. Finally, certain differentiated vertebrate cell types (e.g. neurons, myoblasts) are able to generate specialised Golgi compartments, but the mechanisms have yet to be elucidated $[78,79]$. By addressing these and other issues we are sure to develop a coherent understanding of this most complex organelle, the Golgi apparatus.

\section{Acknowledgements}

I would like to thank Viki Allan and Francis Barr for critically reading the manuscript and the Wellcome Trust, BBSRC and Lowe Syndome Trust for funding. I am especially grateful to Dr Byung Ho-Kang, University of Florida, Dr Brad Marsh, University of Queensland, and Professor Kathryn Howell, University of Colorado for providing images for Figure 3. I apologise to those whose work I could not cite due to space constraints. 


\section{References}

1. Farquhar MG, Palade GE: The Golgi apparatus (complex)-(1954-1981)-from artifact to center stage. J Cell Biol 1981, 91:77s-103s.

2. Farquhar MG, Palade GE: The Golgi apparatus: 100 years of progress and controversy. Trends Cell Biol 1998, 8:2-10.

3. Marsh BJ, Howell KE: The mammalian Golgi--complex debates. Nat Rev Mol Cell Biol 2002, 3:789-795.

4. Emr S, Glick BS, Linstedt AD, Lippincott-Schwartz J, Luini A, Malhotra V, Marsh BJ, Nakano A, Pfeffer SR, Rabouille C, et al.: Journeys through the Golgi--taking stock in a new era. J Cell Biol 2009, 187:449-453.

5. Glick BS, Nakano A: Membrane traffic within the Golgi apparatus. Annu Rev Cell Dev Biol 2009, 25:113-132.

6. Nakano A, Luini A: Passage through the Golgi. Curr Opin Cell Biol 2010, 22:In press.

7. Orci L, Ravazzola M, Volchuk A, Engel T, Gmachl M, Amherdt M, Perrelet A, Sollner TH, Rothman JE: Anterograde flow of cargo across the golgi stack potentially mediated via bidirectional "percolating" COPI vesicles. Proc Natl Acad Sci U S A 2000, 97:10400-10405.

8. Trucco A, Polishchuk RS, Martella O, Di Pentima A, Fusella A, Di Giandomenico D, San Pietro E, Beznoussenko GV, Polishchuk EV, Baldassarre M, et al.: Secretory traffic triggers the formation of tubular continuities across Golgi sub-compartments. Nat Cell Biol 2004, 6:1071-1081. 
9. Marsh BJ, Volkmann N, McIntosh JR, Howell KE: Direct continuities between cisternae at different levels of the Golgi complex in glucose-stimulated mouse islet beta cells. Proc Natl Acad Sci U S A 2004, 101:5565-5570.

10. Patterson GH, Hirschberg K, Polishchuk RS, Gerlich D, Phair RD, Lippincott-Schwartz J: Transport through the Golgi apparatus by rapid partitioning within a two-phase membrane system. Cell 2008, 133:1055-1067.

11. Mollenhauer HH: An Intercisternal Structure in the Golgi Apparatus. J Cell Biol 1965, 24:504-511.

12. Franke WW, Kartenbeck J, Krien S, VanderWoude WJ, Scheer U, Morre DJ: Inter- and intracisternal elements of the Golgi apparatus. A system of membrane-to-membrane cross-links. Z Zellforsch Mikrosk Anat 1972, 132:365-380.

13. Cluett EB, Brown WJ: Adhesion of Golgi cisternae by proteinaceous interactions: intercisternal bridges as putative adhesive structures. J Cell Sci 1992, 103 ( Pt 3):773784.

14. Barr FA, Puype M, Vandekerckhove J, Warren G: GRASP65, a protein involved in the stacking of Golgi cisternae. Cell 1997, 91:253-262.

15. Shorter J, Watson R, Giannakou ME, Clarke M, Warren G, Barr FA: GRASP55, a second mammalian GRASP protein involved in the stacking of Golgi cisternae in a cell-free system. Embo J 1999, 18:4949-4960.

16. Behnia R, Barr FA, Flanagan JJ, Barlowe C, Munro S: The yeast orthologue of GRASP65 forms a complex with a coiled-coil protein that contributes to ER to Golgi traffic. J Cell Biol 2007, 176:255-261.

17. Kondylis V, Spoorendonk KM, Rabouille C: dGRASP localization and function in the early exocytic pathway in Drosophila S2 cells. Mol Biol Cell 2005, 16:4061-4072. 
18. Levi SK, Bhattacharyya D, Strack RL, Austin JR, 2nd, Glick BS: The Yeast GRASP Grh1 Colocalizes with COPII and Is Dispensable for Organizing the Secretory Pathway. Traffic.

19. Slusarewicz P, Nilsson T, Hui N, Watson R, Warren G: Isolation of a matrix that binds medial Golgi enzymes. J Cell Biol 1994, 124:405-413.

20. Sutterlin C, Polishchuk R, Pecot M, Malhotra V: The Golgi-associated protein GRASP65 regulates spindle dynamics and is essential for cell division. Mol Biol Cell 2005, $16: 3211-3222$

21. Puthenveedu MA, Bachert C, Puri S, Lanni F, Linstedt AD: GM130 and GRASP65dependent lateral cisternal fusion allows uniform Golgi-enzyme distribution. Nat Cell Biol 2006, 8:238-248.

22. Feinstein TN, Linstedt AD: GRASP55 regulates Golgi ribbon formation. Mol Biol Cell 2008, 19:2696-2707.

23. Xiang Y, Wang Y: GRASP55 and GRASP65 play complementary and essential roles in Golgi cisternal stacking. J Cell Biol 188:237-251.

** This study uses RNA inteference and quantitative electron microscopy to show that mammalian GRASPs stack Golgi cisternae in vivo. The two GRASPs have complimentary roles in this process, with both required for a fully stacked Golgi. 24. Wang Y, Seemann J, Pypaert M, Shorter J, Warren G: A direct role for GRASP65 as a mitotically regulated Golgi stacking factor. Embo J 2003, 22:3279-3290.

25. Tang D, Yuan H, Wang Y: The role of GRASP65 in Golgi cisternal stacking and cell cycle progression. Traffic 11:827-842.

* See also [26]. Using biochemical and morphological approaches shows that the first PDZ domain of GRASP65 is sufficient to form oligomers that can cross-link Golgi 
membranes.

26. Sengupta D, Truschel S, Bachert C, Linstedt AD: Organelle tethering by a homotypic PDZ interaction underlies formation of the Golgi membrane network. J Cell Biol 2009, 186:41-55.

* Describes an assay to monitor membrane tethering by GRASP65 by targeting it to mitochondria. Shows that the first PDZ domain of GRASP65 undergoes a novel homotypic interaction allowing formation of trans-oligomers that crossmembranes

27. Bachert C, Linstedt AD: Dual anchoring of the GRASP membrane tether promotes trans pairing. J Biol Chem 285:16294-16301.

28. Barr FA, Nakamura N, Warren G: Mapping the interaction between GRASP65 and GM130, components of a protein complex involved in the stacking of Golgi cisternae. Embo J 1998, 17:3258-3268.

29. Ramirez IB, Lowe M: Golgins and GRASPs: holding the Golgi together. Semin Cell Dev Biol 2009, 20:770-779.

30. Shorter J, Warren G: A role for the vesicle tethering protein, p115, in the post-mitotic stacking of reassembling Golgi cisternae in a cell-free system. J Cell Biol 1999, $146: 57-70$

31. Staehelin LA, Kang BH: Nanoscale architecture of endoplasmic reticulum export sites and of Golgi membranes as determined by electron tomography. Plant Physiol 2008, $147: 1454-1468$

** Describes electron tomography of the plant Golgi highlighting its key structural features. The matrix surrounding Golgi stacks is illustrated as are the numerous intraGolgi vesicles present at the cisternal rims, apparently embedded within the matrix. 
32. Faso C, Boulaflous A, Brandizzi F: The plant Golgi apparatus: last 10 years of answered and open questions. FEBS Lett 2009, 583:3752-3757.

33. Kuo A, Zhong C, Lane WS, Derynck R: Transmembrane transforming growth factoralpha tethers to the PDZ domain-containing, Golgi membrane-associated protein p59/GRASP55. Embo J 2000, 19:6427-6439.

* See [34].

34. D'Angelo G, Prencipe L, Iodice L, Beznoussenko G, Savarese M, Marra P, Di Tullio G, Martire G, De Matteis MA, Bonatti S: GRASP65 and GRASP55 sequentially promote the transport of C-terminal valine-bearing cargos to and through the Golgi complex. J Biol Chem 2009, 284:34849-34860.

* Together with [33] shows that GRASPs bind directly to certain cargo proteins as they as they transit the secretory pathway. Binding is required for efficient trafficking, highlighting a novel role for GRASPs in membrane traffic.

35. Kinseth MA, Anjard C, Fuller D, Guizzunti G, Loomis WF, Malhotra V: The Golgiassociated protein GRASP is required for unconventional protein secretion during development. Cell 2007, 130:524-534.

36. Schotman H, Karhinen L, Rabouille C: dGRASP-mediated noncanonical integrin secretion is required for Drosophila epithelial remodeling. Dev Cell 2008, 14:171182.

37. Duran JM, Anjard C, Stefan C, Loomis WF, Malhotra V: Unconventional secretion of Acb1 is mediated by autophagosomes. J Cell Biol 188:527-536.

* Follows on from earlier studies ([35] and [36]) showing that GRASP participates in unconventional secretion. Together with [38] this study uncovers a role for yeast GRASP in unconventional secretion via an autophagosomal intermediate. How GRASP 


\section{carries out this function is currently unclear.}

38. Manjithaya R, Anjard C, Loomis WF, Subramani S: Unconventional secretion of Pichia pastoris Acb1 is dependent on GRASP protein, peroxisomal functions, and autophagosome formation. J Cell Biol 188:537-546.

* See [37].

39. Itoh T, Fujita N, Kanno E, Yamamoto A, Yoshimori T, Fukuda M: Golgi-resident small GTPase Rab33B interacts with Atg16L and modulates autophagosome formation. Mol Biol Cell 2008, 19:2916-2925.

40. Yen WL, Shintani T, Nair U, Cao Y, Richardson BC, Li Z, Hughson FM, Baba M, Klionsky DJ: The conserved oligomeric Golgi complex is involved in doublemembrane vesicle formation during autophagy. J Cell Biol 188:101-114.

41. Zoppino FC, Militello RD, Slavin I, Alvarez C, Colombo MI: Autophagosome formation depends on the small GTPase Rab1 and functional ER exit sites. Traffic.

42. Mowbrey K, Dacks JB: Evolution and diversity of the Golgi body. FEBS Lett 2009, $583: 3738-3745$.

43. Mogelsvang S, Marsh BJ, Ladinsky MS, Howell KE: Predicting function from structure: 3D structure studies of the mammalian Golgi complex. Traffic 2004, 5:338-345.

44. Burkhardt JK: The role of microtubule-based motor proteins in maintaining the structure and function of the Golgi complex. Biochim Biophys Acta 1998, 1404:113-126.

45. Cole NB, Sciaky N, Marotta A, Song J, Lippincott-Schwartz J: Golgi dispersal during microtubule disruption: regeneration of Golgi stacks at peripheral endoplasmic reticulum exit sites. Mol Biol Cell 1996, 7:631-650. 
46. Chabin-Brion K, Marceiller J, Perez F, Settegrana C, Drechou A, Durand G, Pous C: The Golgi complex is a microtubule-organizing organelle. Mol Biol Cell 2001, 12:20472060.

47. Efimov A, Kharitonov A, Efimova N, Loncarek J, Miller PM, Andreyeva N, Gleeson P, Galjart N, Maia AR, McLeod IX, et al.: Asymmetric CLASP-dependent nucleation of noncentrosomal microtubules at the trans-Golgi network. Dev Cell 2007, 12:917-930.

** Compelling live cell imaging shows the nucleation of microtubules at the Golgi apparatus. Microtubule polymerization is dependent upon recruitment of the regulator CLASP to the Golgi by the golgin GCC185. See also [48] and [49]

48. Rivero S, Cardenas J, Bornens M, Rios RM: Microtubule nucleation at the cis-side of the Golgi apparatus requires AKAP450 and GM130. Embo J 2009, 28:1016-1028.

* Together with [47] and [49] shows Golgi-nucleation of microtubules, although the mechanism of nucleation is different in this study, requiring AKAP450 recruitment to the Golgi by GM130. As in [47] and [49] the Golgi-nucleated microtubules are orientated towards the leading edge and required for polarised secretion.

49. Miller PM, Folkmann AW, Maia AR, Efimova N, Efimov A, Kaverina I: Golgi-derived CLASP-dependent microtubules control Golgi organization and polarized trafficking in motile cells. Nat Cell Biol 2009, 11:1069-1080.

* Following on from [47] this study shows that Golgi-nucleated microtubules are required for polarised secretion, in agreement with [48]. It also shows these microtubules play an important role in the assembly and maintenance of the Golgi ribbon.

50. Seemann J, Jokitalo E, Pypaert M, Warren G: Matrix proteins can generate the higher order architecture of the Golgi apparatus. Nature 2000, 407:1022-1026. 
51. Lucocq JM, Pryde JG, Berger EG, Warren G: A mitotic form of the Golgi apparatus in HeLa cells. J Cell Biol 1987, 104:865-874.

52. Mogelsvang S, Gomez-Ospina N, Soderholm J, Glick BS, Staehelin LA: Tomographic evidence for continuous turnover of Golgi cisternae in Pichia pastoris. Mol Biol Cell 2003, 14:2277-2291.

53. Sinka R, Gillingham AK, Kondylis V, Munro S: Golgi coiled-coil proteins contain multiple binding sites for Rab family G proteins. J Cell Biol 2008, 183:607-615.

54. Donohoe BS, Kang BH, Staehelin LA: Identification and characterization of COPIa- and COPIb-type vesicle classes associated with plant and algal Golgi. Proc Natl Acad Sci US A 2007, 104:163-168.

55. Orci L, Perrelet A, Rothman JE: Vesicles on strings: morphological evidence for processive transport within the Golgi stack. Proc Natl Acad Sci U S A 1998, 95:22792283.

56. Ward TH, Polishchuk RS, Caplan S, Hirschberg K, Lippincott-Schwartz J: Maintenance of Golgi structure and function depends on the integrity of ER export. $J$ Cell Biol 2001, 155:557-570.

57. Short B, Haas A, Barr FA: Golgins and GTPases, giving identity and structure to the Golgi apparatus. Biochim Biophys Acta 2005, 1744:383-395.

58. Barr FA, Lambright DG: Rab GEFs and GAPs. Curr Opin Cell Biol 2010, 22:In press.

59. Bisel B, Wang Y, Wei JH, Xiang Y, Tang D, Miron-Mendoza M, Yoshimura S, Nakamura N, Seemann J: ERK regulates Golgi and centrosome orientation towards the leading edge through GRASP65. J Cell Biol 2008, 182:837-843.

* This study shows that phosphorylation of GRASP65 by ERK is required for Golg repositioning towards the leading edge of migrating cells, most likely by remodelling the 


\section{Golgi matrix. Interestingly, centrosome repositioning also requires GRASP}

\section{phosphorylation, consistent with a functional coupling of these structures.}

60. Lowe M, Barr FA: Inheritance and biogenesis of organelles in the secretory pathway. Nat Rev Mol Cell Biol 2007, 8:429-439.

61. Wu CC, MacCoss MJ, Mardones G, Finnigan C, Mogelsvang S, Yates JR, 3rd, Howell KE: Organellar proteomics reveals Golgi arginine dimethylation. Mol Biol Cell 2004, 15:2907-2919.

62. Beck KA: Spectrins and the Golgi. Biochim Biophys Acta 2005, 1744:374-382.

63. Egea G, Lazaro-Dieguez F, Vilella M: Actin dynamics at the Golgi complex in mammalian cells. Curr Opin Cell Biol 2006, 18:168-178.

64. Brownhill K, Wood L, Allan V: Molecular motors and the Golgi complex: staying put and moving through. Semin Cell Dev Biol 2009, 20:784-792.

65. Dippold HC, Ng MM, Farber-Katz SE, Lee SK, Kerr ML, Peterman MC, Sim R, Wiharto PA, Galbraith KA, Madhavarapu S, et al.: GOLPH3 bridges phosphatidylinositol-4phosphate and actomyosin to stretch and shape the Golgi to promote budding. Cell 2009, 139:337-351.

66. Kondylis V, van Nispen tot Pannerden HE, Herpers B, Friggi-Grelin F, Rabouille C: The golgi comprises a paired stack that is separated at G2 by modulation of the actin cytoskeleton through Abi and Scar/WAVE. Dev Cell 2007, 12:901-915.

67. Kondylis V, Rabouille C: The Golgi apparatus: lessons from Drosophila. FEBS Lett 2009, 583:3827-3838.

68. Rothman JE: The golgi apparatus: two organelles in tandem. Science 1981, 213:12121219. 
69. Wang Y, Wei JH, Bisel B, Tang D, Seemann J: Golgi cisternal unstacking stimulates COPI vesicle budding and protein transport. PLoS One 2008, 3:e1647.

70. Yadav S, Puri S, Linstedt AD: A primary role for Golgi positioning in directed secretion, cell polarity, and wound healing. Mol Biol Cell 2009, 20:1728-1736.

71. Sutterlin C, Hsu P, Mallabiabarrena A, Malhotra V: Fragmentation and dispersal of the pericentriolar Golgi complex is required for entry into mitosis in mammalian cells. Cell 2002, 109:359-369.

72. Colanzi A, Corda D: Mitosis controls the Golgi and the Golgi controls mitosis. Curr Opin Cell Biol 2007, 19:386-393.

73. Kodani A, Sutterlin C: The Golgi protein GM130 regulates centrosome morphology and function. Mol Biol Cell 2008, 19:745-753.

74. Altan-Bonnet N, Sougrat R, Lippincott-Schwartz J: Molecular basis for Golgi maintenance and biogenesis. Curr Opin Cell Biol 2004, 16:364-372.

75. Drin G, Morello V, Casella JF, Gounon P, Antonny B: Asymmetric tethering of flat and curved lipid membranes by a golgin. Science 2008, 320:670-673.

** Elegant study showing that the golgin GMAP210 is sufficient to tether membranes in vitro. Tethering is by each end of the protein, and is asymmetric, suggesting a mechanism for capturing transport vesicles by Golgi cisternae.

76. Hennies HC, Kornak U, Zhang H, Egerer J, Zhang X, Seifert W, Kuhnisch J, Budde B, Natebus M, Brancati F, et al.: Gerodermia osteodysplastica is caused by mutations in SCYL1BP1, a Rab-6 interacting golgin. Nat Genet 2008, 40:1410-1412.

* Together with [77] provides evidence that golgin-mediated tethering is important in the context of human physiology and that loss of a single golgin can lead to disease. In this study mutation of the golgin SCYL1BP1 is described. 
77. Smits P, Bolton AD, Funari V, Hong M, Boyden ED, Lu L, Manning DK, Dwyer ND, Moran JL, Prysak M, et al.: Lethal skeletal dysplasia in mice and humans lacking the golgin GMAP-210. N Engl J Med 362:206-216.

* Shows that mutation of the golgin GMAP210 impairs proteoglycan secretion in mammals, causing disease. Together with [76[ indicates physiological importance of golgins.

78. Hanus C, Ehlers MD: Secretory outposts for the local processing of membrane cargo in neuronal dendrites. Traffic 2008, 9:1437-1445.

79. Lu Z, Joseph D, Bugnard E, Zaal KJ, Ralston E: Golgi complex reorganization during muscle differentiation: visualization in living cells and mechanism. Mol Biol Cell 2001, 12:795-808.

\section{Figure Legends}

Figure 1 Model for GRASP function in Golgi stacking and ribbon formation. A, Golgi stacking. During Golgi assembly or cisternal maturation newly forming cisternae at the cisside of the Golgi apparatus are first tethered to the cis-Golgi via long-range golgin-mediated attachment. This allows GRASP65 on both compartments to interact via PDZ1-mediated trans-oligomerization, resulting in cross-linking and stacking of the cisternae. GRASP55 trans-oligomers are responsible for cross-linking later cisternae in the stack.. B, Golgi ribbon formation. Here, cisternae are laterally connected via golgins, leading to the transoligomerization of GRASP65 and 55 at the rims of the cis and medial cisternae respectively. This leads to membrane fusion between the cisternae and Golgi ribbon formation. 
Figure 2 The role of microtubules in Golgi ribbon formation and polarized secretion. A, Assembly of the Golgi ribbon occurs in 2 phases. The first phase involves nucleation and polymerization of microtubules at Golgi stacks. The microtubules act in a 'search and capture' manner to contact other Golgi stacks in the vicinity that move along the microtubules towards the minus end, brining the stacks together in the cell periphery. The clustered stacks move along centrosomally-nucleated microtubules towards the minus ends, bringing them together in the cell centre. Tubular connections are made between the stacks resulting in the formation of a continuous Golgi ribbon (not shown). B, Golgi nucleated microtubules extend towards the leading edge of a migrating cell. TGN-derived transport carriers move along these microtubules towards the plus ends to mediate delivery of new membrane and secretory cargo at the leading edge of the cell. See references [47-49].

Figure 3. Electron tomographic models of the Golgi apparatus. A, Golgi stack from an Arabidopsis thaliana meristem cell. The different cisternae within the stack and the flanking trans-Golgi network (TGN) are colour-labelled. The ribosome excluding Golgi matrix is shown in white. Note how the matrix extends around the surface of the Golgi stack and associated TGN. The blue spheres are clathrin-coated vesicles at the TGN. Image kindly provided by Dr Byung Ho-Kang, University of Florida. Bar, $500 \mathrm{~nm}$. B, Structure of the Golgi ribbon from a hamster pancreatic cell line. The Golgi stacks are laterally arranged within the Golgi ribbon. The cisternae are colour coded, going from cis to trans as indicated. Numerous transport vesicles that surround the Golgi stacks are shown in white. Note the close proximity of the vesicles to the Golgi stacks. Bar, $500 \mathrm{~nm}$. Image from reference [3] with permission. Kindly provided by Dr Brad Marsh, University of Queensland and Professor Kathryn Howell, University of Colorado. 


\section{(A) Stacking of Golgi cisternae}
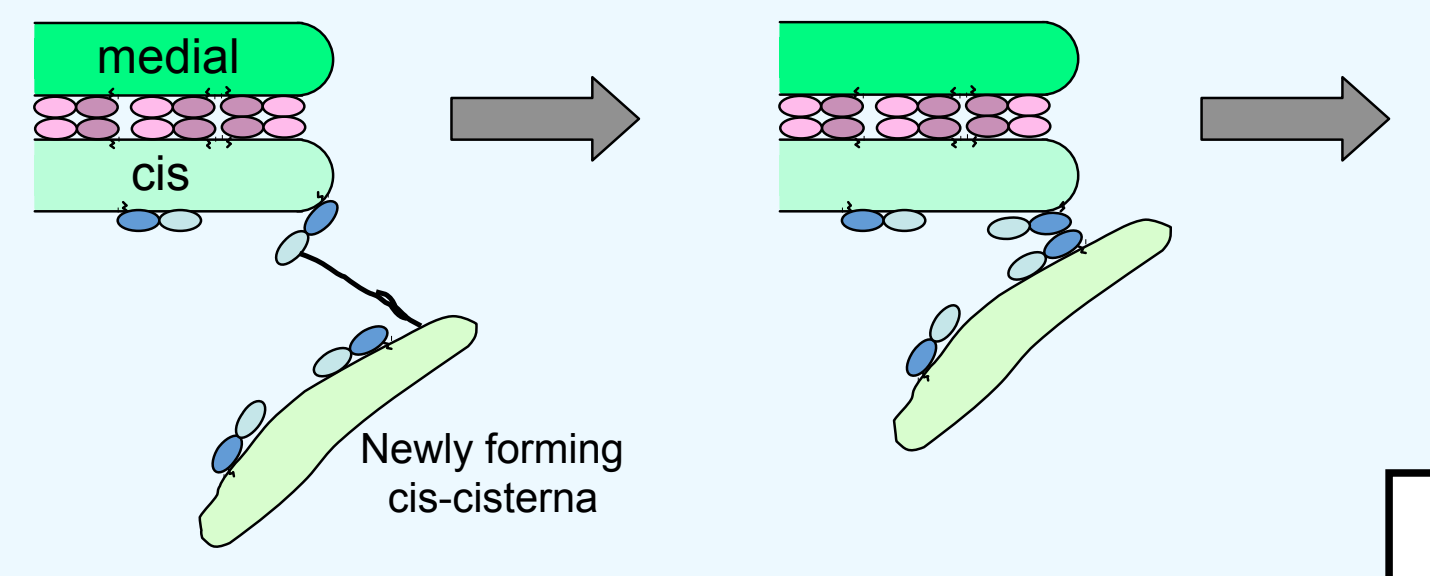

(B) Formation of Golgi ribbon
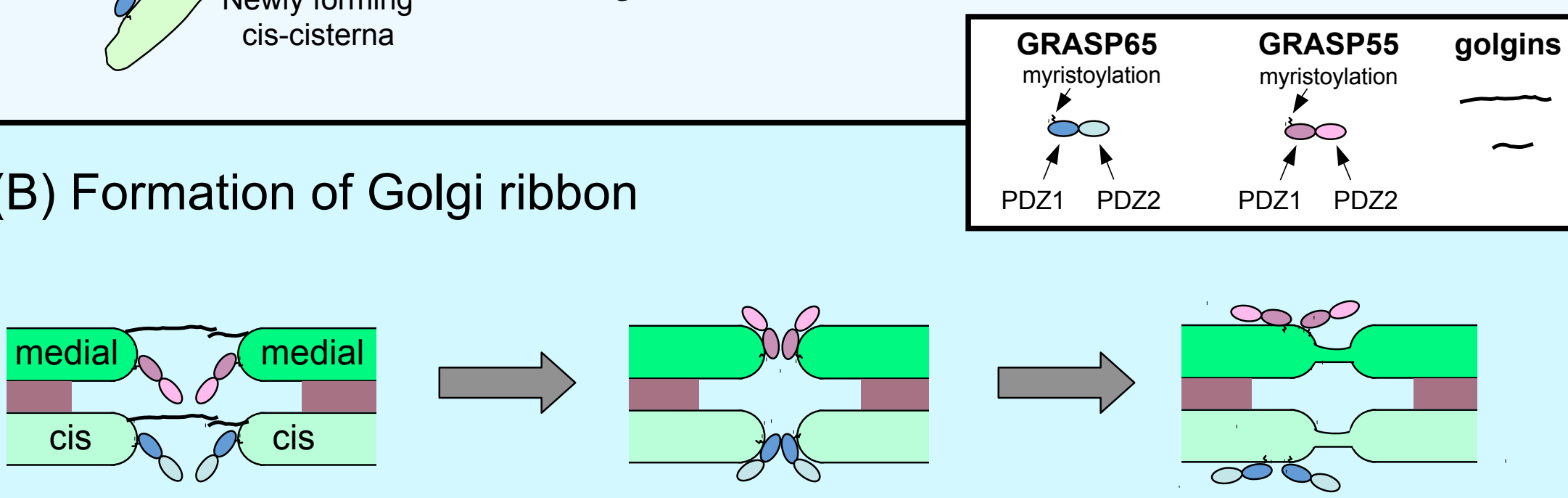

Figure 1 
(A) Golgi ribbon assembly
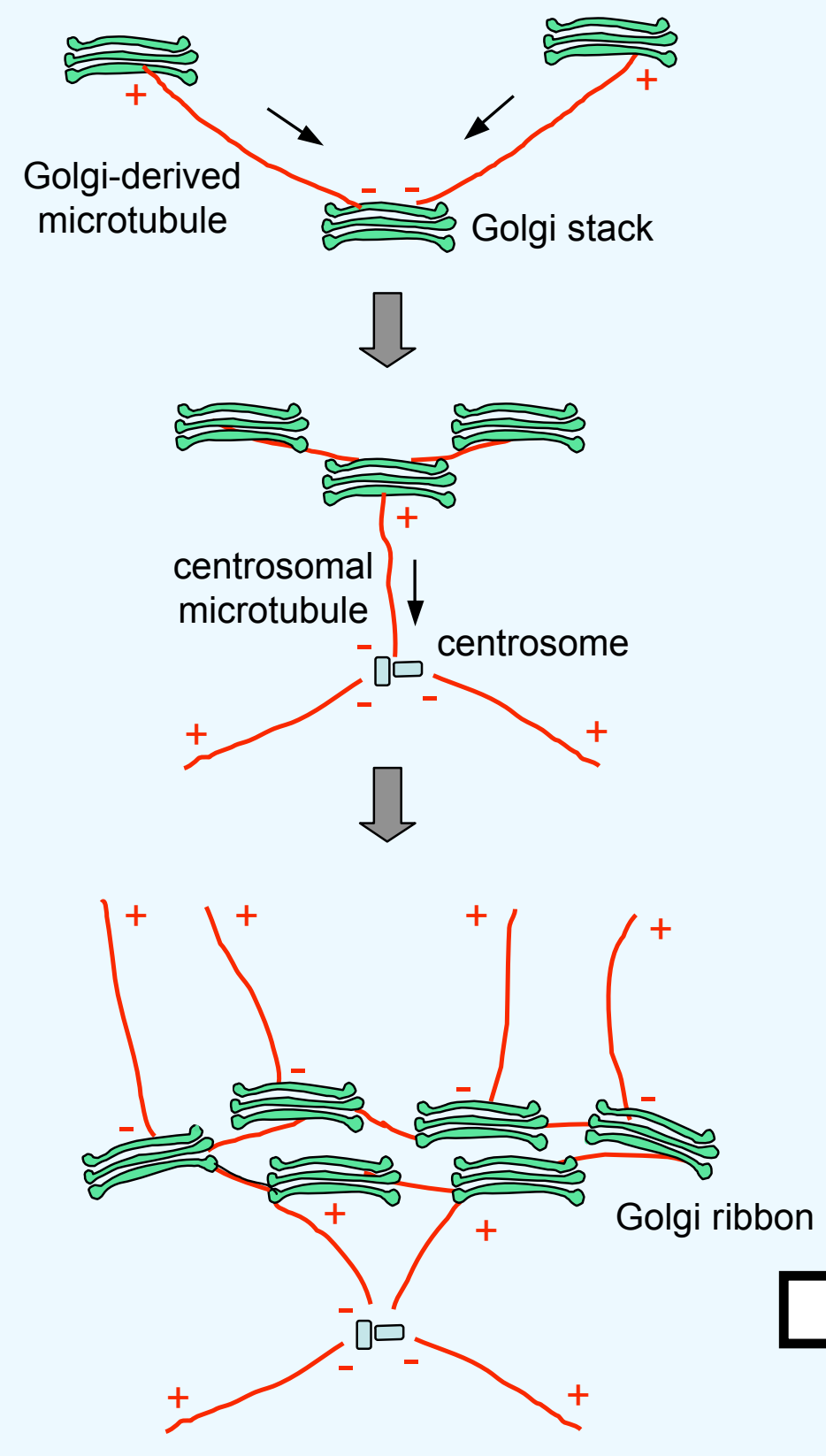

(B) Golgi ribbon for polarised secretion

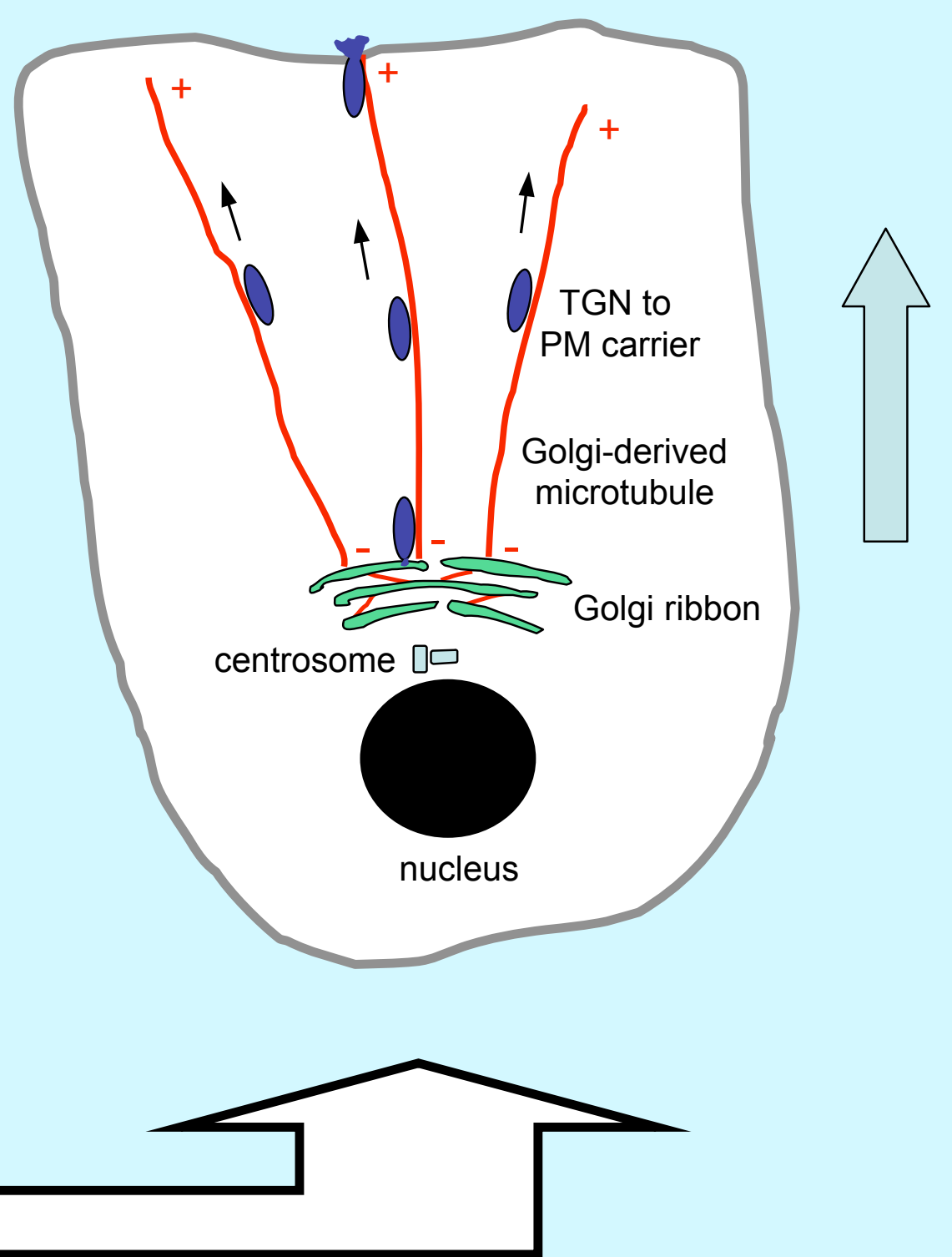

Figure 2 

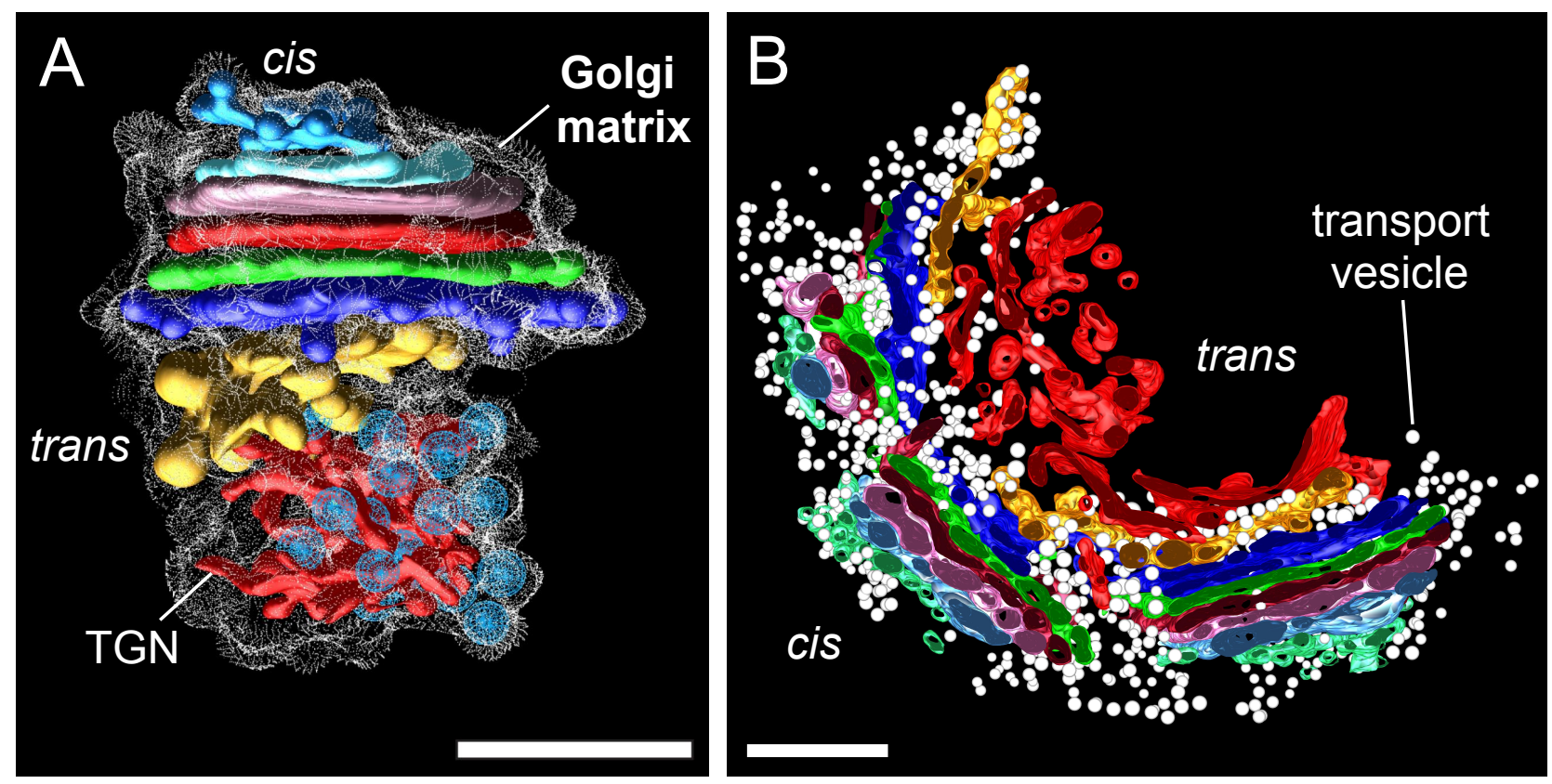

Figure 3 